\title{
A consequência dos polimorfismos genéticos dos fatores de virulência cagA e vacA nas desordens gastroduodenais acometidas por Helicobacter pylori
}

\author{
The consequence of genetic polymorphisms of cagA and vacA virulence factors on gastroduodenal \\ disorders affected by Helicobacter pylori \\ La consecuencia de los polimorfismos genéticos de los factores de virulencia cagA y vacA en los \\ trastornos gastroduodenales afectados por el Helicobacter pylori
}

Recebido: 13/02/2021 | Revisado: 20/02/2021 | Aceito: 01/04/2021 | Publicado: 04/04/2021

Cleiton Moreira Santana

ORCID: https://orcid.org/0000-0001-6978-3531

Faculdade Nobre de Feira de Santana, Brasil E-mail:bm.cleitonmoreira@gmail.com

Raíssa Lima Santos

ORCID: https://orcid.org/0000-0001-7087-5484

Faculdade Nobre de Feira de Santana, Brasil E-mail: bioraissalima@outlook.com

Tarciso De Figueiredo Palma

ORCID: https://orcid.org/0000-0002-3738-8761 Unidade de Ensino Superior de Feira de Santana, Brasil E-mail: tfpalma@gmail.com

Ana Carolina Santana De Oliveira

ORCID: https://orcid.org/0000-0001-8335-2359

Unidade de Ensino Superior de Feira de Santana, Brasil E-mail: anasantanoli@yahoo.com

\begin{abstract}
Resumo
Os fatores de virulência cagA e vacA, estão intimamente relacionados a patogenicidade da Helicobacter pylori. Estes fatores são amplamente estudados e associados com um maior risco de desenvolvimento de diversos desfechos clínicos gastroduodenais, como o câncer gástrico. Além de apresentarem diversos polimorfismos genéticos, que podem variar de acordo com a região geográfica, acarretando em diferentes níveis de toxicidade e manifestações clínicas gástricas. Através de uma revisão integrativa da literatura analisou-se os estudos clínicos/epidemiológicos publicados entre 2015 e 2020, nas bases de dados LILACS, Scielo e PubMed, objetivando reunir os dados mais recentes e importantes sobre os diversos polimorfismos nos fatores de virulência cagA e vacA e os desfechos clínicos gastroduodenais. Foram identificados 19 estudos que contemplavam o objetivo desta revisão e foi observada uma grande variedade de polimorfismos e combinações genéticas destes fatores de virulência em associação com diversos desfechos clínicos. Notou-se também, a existência de perfis genéticos de prevalência das cepas de $H$. pylori e associações com as patologias gástricas distintas em certas regiões geográficas. Apesar de identificados alguns resultados conflitantes, a compreensão destes achados e a identificação dos possíveis genes contribuintes nas patologias gástricas, podem servir como marcadores para o desenvolvimento de estratégias para a prevenção dessas patologias e melhor eficácia do tratamento.
\end{abstract}

Palavras-chave: Helicobacter pylori; CagA; VacA; Gastropatias.

\begin{abstract}
The virulence factors cagA and vacA, are closely related to the pathogenicity of Helicobacter pylori. These factors are widely studied and associated with a higher risk of developing several gastroduodenal clinical outcomes, such as gastric cancer. Besides presenting several genetic polymorphisms, which can vary according to the geographic region, leading to different levels of toxicity and clinical gastric manifestations. Through an integrative review of the literature we analyzed the clinical/epidemiological studies published between 2015 and 2020 in the LILACS, Scielo and PubMed databases, aiming to gather the most recent and important data on the various polymorphisms in the factors of virulence cagA and vacA and gastroduodenal clinical outcomes. Nineteen studies were identified that contemplated the objective of this review and a great variety of polymorphisms and genetic combinations of these virulence factors were observed in association with several clinical outcomes. It was also noted the existence of genetic profiles of prevalence of $H$. pylori strains and associations with distinct gastric pathologies in certain geographical regions. Although some conflicting results were identified, the understanding of these findings and the identification of possible contributing genes in gastric pathologies may serve as markers for the development of
\end{abstract}


strategies for the prevention of these pathologies and better treatment efficacy.

Keywords: Helicobacter pylori; Virulence factors; CagA; VacA; Gastropathies.

\section{Resumen}

Los factores de virulencia cagA y vacA están estrechamente relacionados con la patogenicidad de Helicobacter pylori. Estos factores están ampliamente estudiados y se asocian a un mayor riesgo de desarrollar varios resultados clínicos gastroduodenales, como el cáncer gástrico. Además de presentar varios polimorfismos genéticos, que pueden variar según la región geográfica, dando lugar a diferentes niveles de toxicidad y manifestaciones clínicas gástricas. A través de una revisión integradora de la literatura se analizaron los estudios clínicos/epidemiológicos publicados entre 2015 y 2020 en las bases de datos LILACS, Scielo y PubMed, con el objetivo de reunir los datos más recientes e importantes sobre los distintos polimorfismos en los factores de virulencia cagA y vacA y los resultados clínicos gastroduodenales. Se identificaron 19 estudios que abordaron el objetivo de esta revisión y se observó una amplia variedad de polimorfismos y combinaciones genéticas de estos factores de virulencia en asociación con varios resultados clínicos. También se observó la existencia de perfiles genéticos de prevalencia de cepas de $H$. pylori y de asociaciones con distintas patologías gástricas en determinadas regiones geográficas. Aunque se identificaron algunos resultados contradictorios, la comprensión de estos hallazgos y la identificación de los posibles genes que contribuyen a las patologías gástricas pueden servir como marcadores para el desarrollo de estrategias para la prevención de estas patologías y una mejor eficacia del tratamiento.

Palabras clave: Helicobacter pylori; Factores de virulência; CagA, VacA; Gastropatías.

\section{Introdução}

Helicobacter pylori é uma das bactérias mais comuns, capazes de colonizar a mucosa gastroduodenal de seres humanos; estima-se que mais da metade da população mundial está infectada, porém a taxa de infecção varia entre as regiões geográficas (Hooi et al., 2017). Esta bactéria tem sido categorizada como um carcinogênico classe I e está envolvida no desenvolvimento de diversas complicações gastroduodenais, como: gastrite crônica, úlceras pépticas e duodenais, câncer e linfoma gástrico (Hatakeyama 2017; Diaconu, Predescu, Moldoveanu, Pop \& Fierbinteanu-Braticevici, 2017).

A maioria dos indivíduos infectados com essa bactéria são assintomáticos e devido à grande diversidade de manifestações clínicas da infecção por H. pylori, vários fatores contribuintes são propostos como: fatores de virulência bacteriana, fatores ambientais e fatores genéticos do hospedeiro. Os fatores de virulência são os principais contribuintes na patogênese da infecção por $H$. pylori, uma vez que cepas virulentas são muito mais agressivas e com maiores probabilidades de desenvolvimento de manifestações clínicas graves (Diaconu, Predescu, Moldoveanu, Pop \& Fierbinteanu-Braticevici, 2017; Ansari \& Yamaoka, 2019).

O genoma de $H$. pylori possui a capacidade de codificar uma vasta gama de fatores de virulência e um dos mais importantes são o gene A associado a citotoxina (cagA) e a citotoxina vacuolizante (vacA). O cagA tem sido associado à inflamação intensa na mucosa gástrica e a desfechos clínicos gastroduodenais graves, como câncer gástrico, o que o fez ser caracterizado como uma oncoproteína bacteriana (Kao, Sheu \& Wu, 2016; Ansari \& Yamaoka, 2019).

Já vacA é uma exotoxina que apresenta diversos efeitos pleiotróficos, sendo sua principal característica, a capacidade de induzir a vacuolização de suas células hospedeiras. Trata-se de um gene presente em todas as cepas de $H$. pylori, porém com variações na atividade vacuolizante. Isto deve-se a polimorfismos de algumas regiões do gene vacA, como: região-s, região-m, região-i, região-d e a mais recente identificada, região-c (Whitmire \& Merrell, 2019; McClain, Beckett \& Cover, 2017).

Os diversos polimorfismos desses dois fatores de virulência e a associação com as diversas manifestações clínicas da infecção por H. pylori tem sido objeto de várias investigações. Já foram documentados que cepas cagA positivas e os genótipos vacA, principalmente o $\mathrm{s} 1$ e m1, são prevalentes em diversas lesões graves ao epitélio gástrico, como úlceras pépticas e câncer gástrico e também são evidenciados maior grau de inflamação e liberação de citocinas inflamatórias (Vinagre, Queiroz, Silva Júnior, Vinagre \& Martins, 2015; Tserentogtokh et al., 2019; Pajavand et al., 2015).

Partindo deste princípio, objetiva-se através da sistematização dos dados clínicos/epidemiológicos encontrados, 
discutir sobre as variações alélicas mais prevalentes dos fatores de virulência cagA e vacA nas diversas cepas de $H$. pylori e suas implicações nas diversas manifestações gastroduodenais. Espera-se assim, salientar a necessidade da detecção precoce destas cepas virulentas nos indivíduos infectados por $H$. pylori, principalmente os assintomáticos, para uma gestão mais eficiente do paciente.

\section{Metodologia}

\subsection{Tipo de estudo}

O presente artigo utilizou a revisão integrativa da literatura para sintetizar os resultados de estudos sobre o impacto dos polimorfismos genéticos nos fatores de virulência cagA e vacA nos diversos desfechos clínicos gastroduodenais. Este tipo de revisão, de abordagem qualitativa, tem como objetivo avaliar dados de diversos estudos científicos, que possibilitam a redução através da síntese e união de resultados gerais sobre um determinado tema, sua exposição, comparação, bem como na conclusão e verificação dos dados, resultando na produção de novos conhecimentos sobre o assunto escolhido (Souza, Silva \& Carvalho, 2010).

O rigor desta revisão é garantido em função do seguimento criterioso das seguintes etapas: (1) elaboração da hipótese ou questão norteadora da pesquisa; (2) descrição da estratégia de busca bibliográfica e estabelecimento dos critérios de inclusão e exclusão; (3) extração, organização e sumarização das informações (formação do banco de dados); (4) análise e interpretação dos resultados, aplicando os critérios estabelecidos; e (5) apresentação dos resultados e síntese do conhecimento, através de categorias analíticas (Mendes, Silveira \& Galvão, 2008).

\subsection{Questão norteadora}

Com o intuito de guiar a revisão integrativa, foi formulada a seguinte questão norteadora: Quais as consequências dos diversos polimorfismos genéticos nos fatores cagA e vacA nos desfechos clínicos gastroduodenais de pacientes infectados por Helicobacter pylori?

\subsection{Estratégia de busca}

Após a identificação da questão norteadora, foi realizado um levantamento bibliográfico nas seguintes bases de dados: Literatura Latino-Americana e do Caribe em Ciências da Saúde (LILACS), Scientific Eletronic Library Online (SciELO) e na PubMed (National Library of Medicine). A busca foi realizada através dos artigos publicados no período de 2015 a 2020 e mediante a utilização dos seguintes Descritores de Ciências da Saúde (DeCS): "Helicobacter pylori", "cagA", "vacA" e "gastropatias", bem como os respectivos descritores em inglês e espanhol, agrupados com o operador booleano "and".

\subsection{Critérios de inclusão e exclusão}

Foram aplicados critérios de inclusão e exclusão para auxiliar na seleção dos estudos, e só foram incluídos artigos clínicos/epidemiológicos originais sobre as associações dos fatores de virulência do H. pylori (cagA e vacA) nas patologias gástricas, além de artigos publicados nos idiomas português, inglês e espanhol. Foram excluídos os artigos que se apresentavam fora do corte temporal, artigos de revisão, metanálises e revisões sistemáticas, estudos sem a associação dos fatores de virulência de interesse nas patolog2ias gástricas ou em outras patologias.

\subsection{Extração de dados e síntese}

Foi confeccionada uma planilha para sumarizar os artigos, os quais foram selecionados, podendo ser observados 
dados referentes ao estudo como: autores, ano da publicação, base de dado, título do artigo, metodologia e principais resultados encontrados. Posteriormente, estes dados foram analisados para seleção dos artigos que possibilitaram responder o questionamento da presente revisão.

Os artigos foram selecionados a partir da utilização dos descritores e dos critérios de inclusão e exclusão já estabelecidos. Para seleção dos estudos foi realizada primeiramente uma leitura dos títulos com posterior exclusão dos estudos que se repetiam entre as bases (duplicadas). Após isso, foi feita uma leitura dos resumos selecionados e exclusão dos que estavam fora da temática da pesquisa e dos critérios estabelecidos. Por fim, foi realizada uma leitura minuciosa dos estudos restantes e seleção dos que se enquadravam nos critérios de exclusão e inclusão estabelecidos. Um fluxograma foi confeccionado ilustrando todo o processo de seleção e número de estudos em cada fase, Figura 1.

Com intuito de facilitar a observação e a compreensão durante a apresentação da discussão e resultados, os dados considerados mais importantes foram apresentados com auxílio de um quadro (Quadro 1).

Figura 1 - Fluxograma de pesquisa e triagem da literatura.
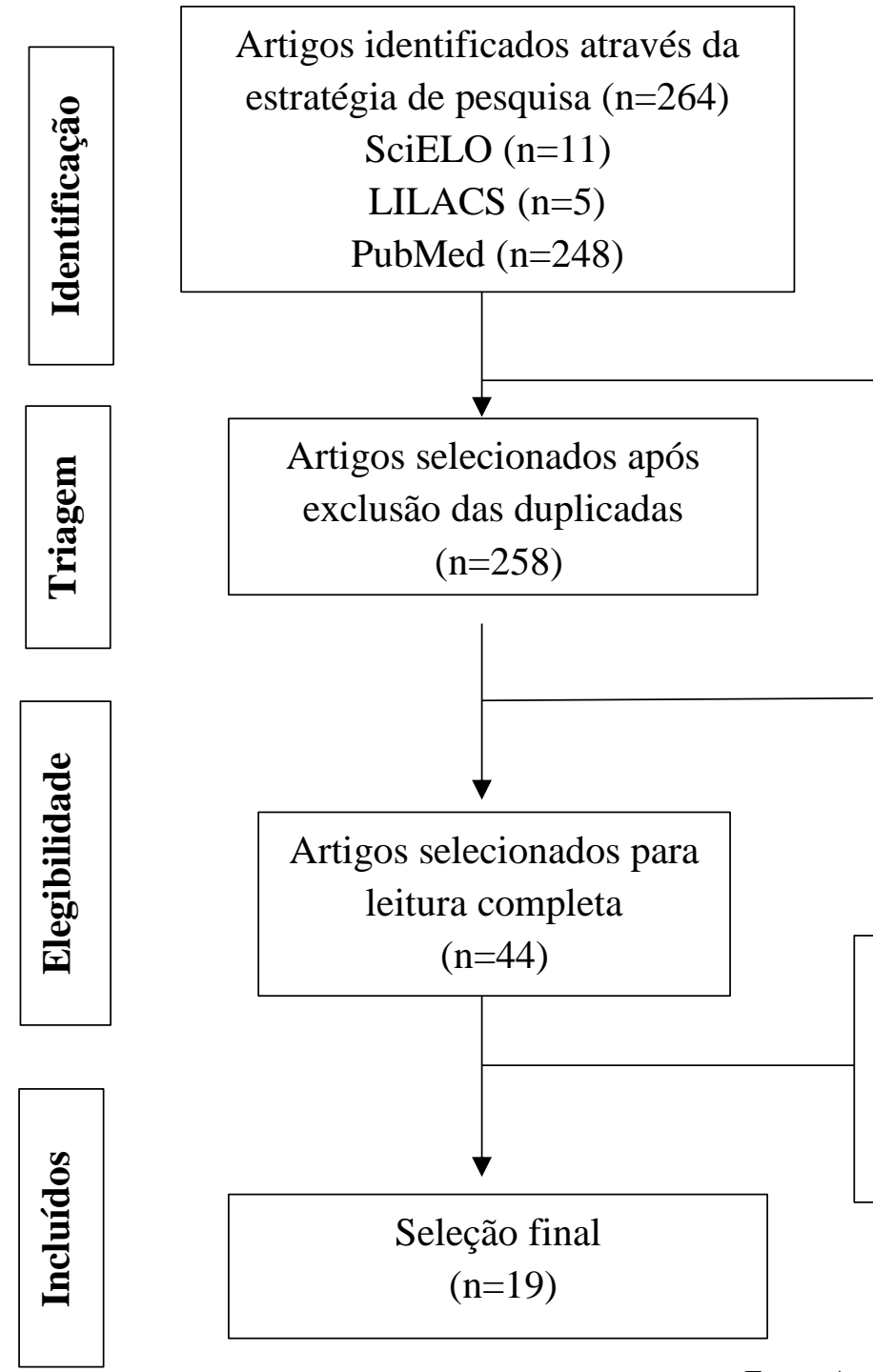

Artigos excluídos ( $\mathrm{n}=6)$

Razão: Artigos duplicados

Fonte: Autores (2021).

\section{Artigos excluídos ( $\mathrm{n}=25)$}

Razão: Não relevante após leitura integral do texto e fora dos critérios estabelecidos. 


\section{Resultados e Discussão}

Através da estratégia de busca inicial nas bases de dados, 264 artigos foram identificados, sendo: Onze (11) SciELO, cinco (5) LILACS e duzentos e quarenta e oito (248) PubMed. Foram excluídos 6 artigos por estarem duplicados e após esta etapa com os 258 artigos restantes, foi realizada uma triagem através da leitura dos títulos e dos resumos, onde foram excluídos 214 artigos por não contemplarem a temática que constitui o objetivo dessa revisão. Dos 44 artigos restantes, após a leitura dos textos na íntegra, foram excluídos 25 devido ao fato de não se enquadrarem aos critérios de inclusão e exclusão estabelecidos, sobrando assim os 19 artigos utilizados no presente estudo. Todo o processo de seleção pode ser observado no fluxograma da figura 1 e a estruturação dos achados no item 3.1 - Caracterização do material do estudo.

\subsection{Caracterização do material do estudo}

No total, 19 artigos foram utilizados para as análises; no que se refere à origem, 18 artigos são internacionais (Cuba, Colômbia, Bangladesh, dois da Arábia Saudita, Gana, Marrocos, África do Sul, Suíça, Japão, Costa Rica, três do Irã, Chile, China, Polônia e Mongólia) e um, nacional, elaborado no estado do Pará. Estes achados nos mostram populações e regiões geográficas distintas e consequentemente variações e associações diferentes dos fatores de virulência cagA e vacA nas infecções por H. pylori.

Identificou-se maior prevalência de publicações no ano de 2017 (7 - 36,8\%), seguido do ano de 2019 (5 - 26,3\%), bem como foram constatadas mais publicações nos periódicos da PloS One (dos Estados Unidos), sendo 3, ou aproximadamente $16 \%$ das publicações. No que tangem as especificidades das revistas, as áreas de Gastrenterologia, Patogêneses e Microbiologia foram as mais escolhidas pelos autores, porém tendo presença significativa, também nos editoriais de ciências médicas em geral.

No que se referem os tipos de estudo, conforme determinado nos fatores de inclusão, todos são estudos epidemiológicos, sendo sua maioria, $13(68,4 \%)$, transversais analíticos, onde foram estimadas medidas de razão de prevalência e odds ratio, variando entre os estudos; e, 6 (31,6\%), meramente descritivos, onde apenas prevalências (frequências) são estimadas de acordo com as características de interesse de cada publicação.

Abaixo segue quadro que apresenta os artigos de acordo com suas características.

Quadro 1. Artigos selecionados.

\begin{tabular}{|c|l|l|l|l|}
\hline País & \multicolumn{1}{|c|}{ Título } & \multicolumn{1}{|c|}{ Autores } & \multicolumn{1}{c|}{ Periódico (Ano) } & \multicolumn{1}{c|}{$\begin{array}{c}\text { Tipo De } \\
\text { Estudo }\end{array}$} \\
\hline IR Ã & $\begin{array}{l}\text { High Frequency of vacA slm2 Genotypes } \\
\text { Among Helicobacter pylori Isolates From } \\
\text { Patients With Gastroduodenal Disorders in } \\
\text { Kermanshah, Iran }\end{array}$ & $\begin{array}{l}\text { Pajavand et al, } \\
2015\end{array}$ & $\begin{array}{l}\text { Jundishapur J Microbiol. } \\
(2015)\end{array}$ & $\begin{array}{l}\text { Transversal } \\
\text { descritivo }\end{array}$ \\
\hline BRASIL & $\begin{array}{l}\text { Helicobacter pylori infection In patients } \\
\text { with different Gastrointestinal diseases from } \\
\text { Northern brazil }\end{array}$ & $\begin{array}{l}\text { Vinagre, } \\
\text { Queiroz, Silva } \\
\text { Júnior, Vinagre } \\
\text { \& Martins, 2015 }\end{array}$ & $\begin{array}{l}\text { Arquivos de } \\
\text { gastroenterologia, (2015) }\end{array}$ & $\begin{array}{l}\text { Transversal } \\
\text { descritivo }\end{array}$ \\
\hline POLÔNIA & $\begin{array}{l}\text { Expression of cagA, virB/D Complex and/or } \\
\text { vacA Genes in Helicobacter pylori Strains } \\
\text { Originating from Patients with Gastric } \\
\text { Diseases }\end{array}$ & $\begin{array}{l}\text { Szkaradkiewicz } \\
\text { et al, 2016 }\end{array}$ & PloS one, (2016) & $\begin{array}{l}\text { Transversal } \\
\text { descritivo }\end{array}$ \\
\hline CHINA & $\begin{array}{l}\text { Helicobacter pylori vacA Genotypes in } \\
\text { Chronic Gastritis and Gastric Carcinoma } \\
\text { Patients from Macau, China }\end{array}$ & $\begin{array}{l}\text { Pinto-Ribeiro et } \\
\text { al, 2016 }\end{array}$ & Toxins, (2016) & $\begin{array}{l}\text { Transversal } \\
\text { analítico }\end{array}$ \\
\hline CUBA & Presencia del gen cagA y de la citotoxina & Leyya, Aleaga, & Revista Cubana de Medicina & Transversal \\
\hline
\end{tabular}


Research, Society and Development, v. 10, n. 4, e28610412938, 2021

(CC BY 4.0) | ISSN 2525-3409 | DOI: http://dx.doi.org/10.33448/rsd-v10i4.12938

\begin{tabular}{|c|c|c|c|c|}
\hline & $\begin{array}{l}\text { vacA del Helicobacter pylori en pacientes } \\
\text { dispépticos }\end{array}$ & $\begin{array}{l}\text { González, } \\
\text { Zamora \& } \\
\text { González, } 2016\end{array}$ & Militar, (2016) & descritivo \\
\hline BANGLADESH & $\begin{array}{l}\text { Two populations of less-virulent } \\
\text { Helicobacter }\end{array}$ & $\begin{array}{l}\text { Aftab et al., } \\
2017\end{array}$ & PloS One, (2017) & $\begin{array}{l}\text { Transversal } \\
\text { analítico }\end{array}$ \\
\hline $\begin{array}{l}\text { ARÁBIA } \\
\text { SAUDITA }\end{array}$ & $\begin{array}{l}\text { Detection and Genotyping of Helicobacter } \\
\text { pylori among Gastric ulcer and Cancer } \\
\text { Patients from Saudi Arabia }\end{array}$ & Bibi et al., 2017 & $\begin{array}{l}\text { Pakistan journal of medical } \\
\text { sciences, (2017) }\end{array}$ & $\begin{array}{l}\text { Transversal } \\
\text { analítico }\end{array}$ \\
\hline GANA & $\begin{array}{l}\text { Helicobacter pylori cagA and vacA genes } \\
\text { in dyspeptic Ghanaian patients }\end{array}$ & $\begin{array}{l}\text { Archampong et } \\
\text { al., } 2017\end{array}$ & $\begin{array}{l}\text { BMC Research Notes, } \\
(2017)\end{array}$ & $\begin{array}{l}\text { Transversal } \\
\text { analítico }\end{array}$ \\
\hline MARROCOS & $\begin{array}{l}\text { VacA and CagA Status as Biomarker of Two } \\
\text { Opposite End Outcomes of Helicobacter } \\
\text { pylori Infection (Gastric Cancer and } \\
\text { Duodenal Ulcer) in a Moroccan Population }\end{array}$ & $\begin{array}{l}\text { El Khadir et al., } \\
2017\end{array}$ & PloS one, (2017) & $\begin{array}{l}\text { Transversal } \\
\text { analítico }\end{array}$ \\
\hline JAPÃO & $\begin{array}{l}\text { Associations between cagA, vacA, and the } \\
\text { clinical outcomes of Helicobacter pylori } \\
\text { infections in Okinawa, Japan }\end{array}$ & $\begin{array}{l}\text { Inagaki et al, } \\
2017\end{array}$ & $\begin{array}{l}\text { The Kobe journal of medical } \\
\text { sciences, (2017) }\end{array}$ & $\begin{array}{l}\text { Transversal } \\
\text { analítico }\end{array}$ \\
\hline CHILE & $\begin{array}{l}\text { Association between cagA, vacAi, and dupA } \\
\text { genes of Helicobacter pylori and } \\
\text { gastroduodenal pathologies in Chilean } \\
\text { patients }\end{array}$ & $\begin{array}{l}\text { Parede-Osses el } \\
\text { al, } 2017\end{array}$ & Folia microbiologica, (2017) & $\begin{array}{l}\text { Transversal } \\
\text { analítico }\end{array}$ \\
\hline IRÃ & $\begin{array}{l}\text { Determination of Helicobacter pylori } \\
\text { virulence-associated genes in duodenal ulcer } \\
\text { and gastric biopsies }\end{array}$ & $\begin{array}{l}\text { Saeidi et al, } \\
2017\end{array}$ & $\begin{array}{l}\text { Medical journal of the } \\
\text { Islamic Republic of } \\
\text { Iran, (2017) }\end{array}$ & $\begin{array}{l}\text { Transversal } \\
\text { descritivo }\end{array}$ \\
\hline IR Ã & $\begin{array}{l}\text { CagA and vacA allelic combination of } \\
\text { Helicobacter pylori in gastroduodenal } \\
\text { Disorders }\end{array}$ & $\begin{array}{l}\text { Sheikh et al, } \\
2018\end{array}$ & $\begin{array}{l}\text { Microbial } \\
\text { pathogenesis, (2018) }\end{array}$ & $\begin{array}{l}\text { Transversal } \\
\text { descritivo }\end{array}$ \\
\hline COLÔMBIA & $\begin{array}{l}\text { Genotyping of } c a g A \text { and the intermediate } \\
\text { region of } v a c A \text { in strains of Helicobacter } \\
\text { pylori isolated from Colombian adult } \\
\text { patients and associations with gastric } \\
\text { diseases }\end{array}$ & $\begin{array}{l}\text { Melo-Narváez } \\
\text { et al., } 2018\end{array}$ & $\begin{array}{l}\text { Rev Colomb Gastroenterol, } \\
\text { (2018) }\end{array}$ & $\begin{array}{l}\text { Transversal } \\
\text { analítico }\end{array}$ \\
\hline $\begin{array}{l}\text { ARÁBIA } \\
\text { SAUDITA }\end{array}$ & $\begin{array}{l}\text { Helicobacter pylori vacA, cagA and iceA } \\
\text { genotypes in dyspeptic patients from } \\
\text { southwestern region, Saudi Arabia: } \\
\text { distribution and association with clinical } \\
\text { outcomes and histopathological changes }\end{array}$ & $\begin{array}{l}\text { Akeel et al., } \\
2019\end{array}$ & $\begin{array}{l}\text { BMC Gastroenterology, } \\
(2019)\end{array}$ & $\begin{array}{l}\text { Transversal } \\
\text { analítico }\end{array}$ \\
\hline $\begin{array}{l}\text { ÁFRICA DO } \\
\text { SUL }\end{array}$ & $\begin{array}{l}\text { Detection of Helicobacter pylori and its } \\
\text { virulence genes (cagA, dupA, and vacA) } \\
\text { among patients with gastroduodenal } \\
\text { diseases in Chris Hani Baragwanath } \\
\text { Academic Hospital, South Africa }\end{array}$ & $\begin{array}{l}\text { Idowu et al., } \\
2019\end{array}$ & $\begin{array}{l}\text { BMC gastroenterology, } \\
\text { (2019) }\end{array}$ & $\begin{array}{l}\text { Transversal } \\
\text { analítico }\end{array}$ \\
\hline SUÍÇA & $\begin{array}{l}\text { Rapid Characterization of Virulence } \\
\text { Determinants in Helicobacter pylori Isolated } \\
\text { from Non-Atrophic Gastritis Patients by } \\
\text { Next-Generation Sequencing }\end{array}$ & $\begin{array}{l}\text { Imkamp et al., } \\
2019\end{array}$ & $\begin{array}{l}\text { Journal of clinical } \\
\text { medicine, (2019) }\end{array}$ & $\begin{array}{l}\text { Transversal } \\
\text { analítico }\end{array}$ \\
\hline COSTA RICA & $\begin{array}{l}\text { Virulence factor genotyping of Helicobacter } \\
\text { pylori isolated from Costa Rican dyspeptic } \\
\text { patients }\end{array}$ & $\begin{array}{l}\text { Molina-Castro, } \\
\text { Garita- } \\
\text { Cambronero, } \\
\text { Malespin- } \\
\text { Bendaña, Une \& } \\
\text { Ramírez, } 2019 \\
\end{array}$ & $\begin{array}{l}\text { Microbial } \\
\text { pathogenesis, (2019) }\end{array}$ & $\begin{array}{l}\text { Transversal } \\
\text { analítico }\end{array}$ \\
\hline MONGOLIA & $\begin{array}{l}\text { Western-Type Helicobacter pylori CagA are } \\
\text { the Most Frequent Type in Mongolian } \\
\text { Patients }\end{array}$ & $\begin{array}{l}\text { Tserentogtokh } \\
\text { et al, } 2019\end{array}$ & Cancers, (2019) & $\begin{array}{l}\text { Transversal } \\
\text { analítico }\end{array}$ \\
\hline
\end{tabular}

Fonte: Autores (2021).

No Quadro 1, foi apresentado a caracterização dos estudos selecionados, segundo o título, delineamento 
metodológico, país de origem, autores e periódico de publicação. É possível observar a variabilidade geográfica dos estudos selecionados, permitindo assim uma observação de populações distintas e o interesse de pesquisadores de várias partes do mundo em produzir conhecimento nessa temática, gerando assim evidências científicas que subsidiem estudos e práticas clínicas no diagnóstico e tratamento das patologias associadas a H. pylori.

Para as análises dos resultados dos artigos desta revisão, emergiram categorias de análise concentradas nos fatores de virulência de H.pylori: (a) cagA, (b) vacA, (c) Associação entre cagA e vacA, que são descritas e racionalizadas, a seguir.

\subsection{Fatores de Virulência de H.pylori}

Os fatores de virulência de $H$. pylori são considerados um dos principais contribuintes para sua patogênese pois possibilitam a colonização, indução de inflamação e danos celulares. Sabe-se que estes fatores de virulência estão associados com o desenvolvimento de patologias gástricas graves e que variações genéticas dos mesmos, apresentam perfis distintos de gravidade (Kao, Sheu \& Wu, 2016).

Sabe-se que as diversas cepas de H. pylori variam quanto à sua virulência, e que as cepas mais virulentas estão associadas a um risco maior de desenvolvimento de distúrbios gastroduodenais. Dentre os inúmeros fatores de virulência deste patógeno destacam-se como os mais frequentes o cagA e o vacA (Sheikh et al., 2018).

Todos os artigos que compõem esta revisão, apresentaram dados referentes aos fatores de virulência cagA e vacA e sua contribuição dos desfechos clínicos gastroduodenais. Para o fator de virulência cagA, foram encontrados seis estudos, que relataram que pacientes infectados por cepas cagA-positivas apresentavam um maior risco de desenvolvimento de patologias mais graves (por exemplo; câncer gástrico) e processos inflamatórios. Também foi observado que as cepas variam geograficamente e as cepas do leste asiático são as mais patogênicas e com possível maior risco de desenvolvimento de câncer gástrico (Aftab et al, 2017; Inagaki et al, 2017; Molina-Castro et al, 2019; Saeidi et al, 2017; Sheikh et al, 2018; Tserentogtokh et al, 2019).

Já para o fator de virulência vacA, foi observado que este apresenta uma grande variação alélica e que expressa diferentes perfis de toxicidade, acarretando em níveis distintos de gravidade da doença. O genótipo vacA s1/m1 foi o mais observado entre os autores, visto em 5 artigos, e associado a um maior risco de desenvolvimento de patologias gastroduodenais, porém a prevalência deste genótipo variava geograficamente e patogenicamente, sendo relatada em pacientes com câncer gástrico, úlceras pépticas, gastrite crônicas à gastrites leves (Narvaez et al, 2018; Leyva et al, 2016; Khadir et al 2017; Inagaki et al, 2017; Tserentogtokh et al, 2019).

No que se refere as associações dos fatores de virulência cagA, vacA e seus subtipos, três estudos relataram a prevalência da associação cagA-positivo e vacA s1m1 em pacientes com câncer gástrico, sendo possível a contribuição dessa cepa para a alta taxa de câncer gástrico no Leste Asiático (Tserentogtokh et al, 2019; Imkamp et al, 2017; Pinto-Ribeiro et al, 2016). A respeito de pacientes diagnosticados com gastrite crônica também foi predominante a associação cagA-positivo e vacA s1m1 em dois estudos (Sheikh et al, 2018; Pinto-Ribeiro et al, 2016).

\subsection{1 cagA}

O cagA é codificado na ilha de patogenicidade de genes associada à citotoxina (cagPAI), trata-se de uma região cromossômica com aproximadamente 40kb, que também codifica o sistema de secreção tipo IV cag (cag-T4SS). O cagA é uma proteína de 120-145kDa que é translocada nas células gástricas através do cag-T4SS, onde irá modular múltiplas cascatas de sinalização associadas à polaridade celular, ciclo celular, proliferação celular, interrupção de junções apertadas e aderentes, respostas pró-inflamatórias levando a inflamações crônicas da mucosa gástrica e indução de carcinogênese, devido supressão 
da apoptose (Backert \& Blaser, 2016; Sterbenc, Jarc, Poljak \& Homan, 2019).

O cagA é um dos fatores de virulência mais amplamente estudados, e as cepas de $H$. pylori são divididas em dois grupos: cagA negativa e cagA positiva. Nos estudos selecionados foi possível observar que a prevalência do gene cagA varia muito conforme as regiões geográficas. Em um estudo no Marrocos a prevalência foi de 61,2\% (El Khadir et al., 2017), na África do Sul obteve-se um resultado semelhante com a prevalência foi de 62\% (Idowu et al., 2019). Resultados inferiores puderam ser observados no estudo da Arábia Saudita com prevalência de 49,2\% (Akeel, et al., 2019) e na Colômbia com prevalência de 48,1\% (Melo-Narvaez et al., 2018), porém em um estudo no Brasil o gene cagA foi identificado em 85,6\% do material isolado (Vinagre, Queiroz, Silva Júnior, Vinagre \& Martins, 2015).

A maioria dos estudos que compuseram esta revisão relataram a presença significativa do gene cagA em pacientes infectados com H. pylori e sua associação com diversos desfechos clínicos gastroduodenais como: gastrite (Imkamp et al., 2019), úlceras duodenais (Akeel et al., 2019; Vinagre, Queiroz, Silva Júnior, Vinagre \& Martins, 2015; Aftab et al., 2017; Archampong et al., 2017), úlcera péptica (Pajavand et al., 2015), úlcera gástrica (Saeidi et al., 2017) e câncer gástrico (Szkaradkiewicz et al., 2016; Tserentogtokh et al, 2019).

Szkaradkiewicz et al. (2016), em um estudo realizado na Polônia, observaram que todas as cepas de H. pylori isoladas de pacientes com gastrite atrófica multifocal e câncer gástrico, carregavam o gene cagA, porém diferenciavam quanto à expressão, pois os pacientes com gastrite atrófica multifocal apresentavam uma maior expressão deste gene, já em pacientes com câncer gástrico a expressão era quase três vezes maior.

Em contrapartida, um estudo realizado por Parede-Osses et al. (2017) mostrou uma baixa prevalência de cepas de $H$. pylori cagA positivas na população chilena infectada, o resultado observado não apresentou diferença nos riscos de desenvolvimento de patologias gástricas graves. Ainda, no estudo de Bibi et al. (2017), foi encontrada uma baixa frequência de cagA na população da Arábia Saudita nos grupos de pacientes com câncer gástrico e úlcera gástrica. Estes achados trazem resultados contraditórios com relação aos estudos anteriores, isto pode ser explicado devido as diferenças geográficas onde as pesquisas foram realizadas e ao número pequeno de amostras analisadas. Estes fatores podem ter sido colaboradores para essa contradição, impossibilitando a obtenção de resultados mais consistentes.

Devido às diversas mutações e recombinações genéticas, existe uma grande diversidade genética em H. pylori, podendo ser observada em sua diferença com relação a distribuição geográfica, potencial de virulência e de perfil de associação com os desfechos clínicos gastroduodenais. Isto ocorre, pois, as cepas cagA apresentam polimorfismos na região terminal C do gene cagA nos pontos Glu-Pro-Ile-Tyr-Ala (EPIYA) (Sterbenc, Jarc, Poljak \& Homan, 2019;).

Com base nestes polimorfismos, as cepas podem ser classificadas em ocidentais (contendo EPIYA-A, -B e -C, podendo -C estar repetido múltiplas vezes) e do leste asiático (contendo EPIYA-A, -B e -D). Esta classificação foi feita devido à prevalência destes pontos específicos da EPIYA nestas regiões geográficas. Vale ressaltar que, cepas do tipo leste asiático (EPIYA-D) são consideradas mais virulentas e frequentemente associadas ao câncer gástrico, entretanto, cepas com múltiplas repetições -C também apresentam o mesmo potencial cancerígeno como em cepas leste asiático (Whitmire \& Merrell, 2019; Tserentogtokh et al., 2019; Idowu et al., 2019).

Aftab et al. (2017) observaram em seu estudo em Bangladesh que a maioria das cepas analisadas eram cagA positivas do tipo ocidental, que foram consideradas como menos virulentas. Em adição, um estudo realizado no Irã, entre as cepas de H.pylori cagA positivas, o cagA do tipo ocidental (EPIYA-ABC e - ABCC) foi observado com mais frequência. O tipo - $\mathrm{ABC}$ foi mais comum nas amostras de câncer gástrico, úlcera péptica e gastroduodenite erosiva, já o -ABCC foi o mais comum em pacientes com dispepsia não ulcerativa (Sheikh et al., 2018).

Tserentogtokh et al. (2019), observaram em seu estudo na Mongólia, que a maioria dos pacientes com câncer gástrico 
foram infectados com o cagA do tipo ocidental. Foi observado também que os danos na mucosa gástrica, principalmente a metaplasia intestinal, foram mais elevados em pacientes infectados com cepas do tipo leste-asiático do que aqueles com cepas cagA do tipo ocidental.

Nesse mesmo estudo, os pacientes infectados com $H$. pylori que apresentavam múltiplas repetições de $-\mathrm{C}$, tinham características histológicas de gastrites mais graves do que aqueles com H. pylori possuindo uma única ou nenhuma repetição de - C. Suportando a ideia de que as cepas do tipo leste asiático ou do tipo ocidental com múltiplas repetições -C são as formas virulentas que causam mais danos a mucosa gástrica (Tserentogtokh et al., 2019).

\subsection{2 vacA}

A exotoxina vacA trata-se de uma molécula com capacidade de induzir a vacuolização da célula hospedeira, apresentando efeitos pleiotrópicos como: despolarização da membrana, autofagia, disfunção mitocondrial, liberação de ânions orgânicos e $\mathrm{HCO}_{3}$ (bicarbonato), ativação de quinases proteicas ativadas por mitogênio, indução de apoptose e inibição da função de células T. Todas estas funções contribuem para a colonização e a patogênese de H. pylori em diversas patologias gástricas (Foegeding, Caston, McClain, Ohi \& Cover, 2018).

Quase todas as cepas de $H$. pylori possuem o gene vacA, porém os diversos polimorfismos alélicos que esta exotoxina apresenta resultam em cepas que produzem vacA com atividade vacuolizante variada. Estas variações estão associadas à combinações de suas cinco regiões já documentadas, como: região s (s1 e s2), região i (i1, i2 e i3), região m (m1 e m2), região d (d1 e d2) e região c (c1 e c2) (Whitmire \& Merrell, 2019; Thi Huven Trang, Thanh Binh \& Yamaoka, 2016).

Como citado anteriormente, sabe-se que quase todas as cepas de H. pylori contêm o gene vacA, da mesma forma no presente estudo foi observado uma grande frequência desse gene nas pesquisas selecionadas. Como o estudo realizado na Arábia Saudita que detectou o gene vacA em 100\% dos isolados (Akeel et al., 2019); no Marrocos foi detectado o gene vacA em 99,06\% dos isolados (El Khadir et al 2017).

As variações alélicas do vacA também diferem significativamente entre cada país e em seus desfechos clínicos gastroduodenais. Dentre os diversos genótipos já conhecidos, a infecção com cepas de $H$. pylori contendo vacA s1/m1 é o mais frequente, afetando cerca de $24-84 \%$ da população mundial e fortemente associada ao aumento do risco de desenvolvimento de patologias gastroduodenais graves (Pajavand et al., 2015).

As infecções por cepas de $H$. pylori com o genótipo $\mathrm{s} 1 / \mathrm{m} 1$ foram frequentemente observados nos estudos realizados: em Gana (Archampong et al., 2017), Costa Rica (Molina-Castro, Garita-Cambronero, Malespin-Bendaña, Une \& Ramírez, 2019), África do Sul (Idowu et al., 2019), Arábia Saudita (Akeel et al., 2019), Brasil (Vinagre, Queiroz, Silva Júnior, Vinagre \& Martins, 2015) e Cuba (Leyya, Aleaga, González, Zamora \& González, 2016). Esse perfil foi o mais prevalente nas amostras, e foi significativamente associado a um risco maior no desenvolvimento de doenças gástricas, como: úlcera duodenal, úlcera péptica, úlcera gástrica, gastrite atrófica e até mesmo câncer gástrico.

Aftab et al. (2017), através de uma análise sequencial indicou que o genótipo vacA predominante das cepas de Bangladesh, vacA s1/m1, esteve associado a maiores atividades inflamatórias e atrofia quando comparado com o genótipo vacA s $2 / \mathrm{m} 2$. Porém, essas regiões do vacA eram do subtipo s1a, um genótipo menos frequente no câncer gástrico, do que em úlceras pépticas e gastrite crônica e a região m foi marcada pelo subtipo m1c, que é específico em países do sul da Ásia com baixa incidência de câncer gástrico.

Um resultado semelhante foi observado por Imkamp et al. (2019) na Suíça, onde a maioria dos isolados de H. pylori carregavam o alelo vacA s1 (56\%), sendo mais frequente o alelo vacA s1a em relação ao alelo s1b. E nos 44\% restantes foram isolados o alelo vacA s2. O genótipo vacA s1/m1, foi considerado o mais tóxico para as células gástricas do que vacA s1/m2, 
sendo associado com úlceras pépticas e um maior risco de desenvolvimento de câncer gástrico. Já as cepas vacA s2/m2, são praticamente não tóxicas e raramente são associadas com patologias gastroduodenais.

Sheikh et al. (2018) observaram que todos os isolados foram vacA positivos e com relação a combinação das regiões s e m, o genótipo vacA s1/m1 foi a combinação alélica mais predominante em pacientes com câncer gástrico e úlcera péptica. Já o genótipo vacA s1/m2 foi a mais frequente combinação alélica nos pacientes com dispepsia não ulcerativa e gastroduodenite erosiva.

Inagaki et al. (2017) observaram em seu estudo em Okinawa, no Japão, que em todas as cepas de $H$. pylori que apresentavam genes vacA do tipo s1/m1 carregavam o gene vacA do tipo i1, e todas as cepas com genes vacA do tipo s2/m2 tinham genes vacA do tipo i2. E quanto às associações com os desfechos clínicos, todas as cepas de H. pylori coletadas dos pacientes com câncer gástrico e úlceras gástricas tinham genes vacA do tipo i1.

Foram observados resultados semelhantes em estudos realizados na China (Pinto-Ribeiro et al., 2016), Chile (ParedeOsses et al., 2017), Bangladesh (Aftab et al., 2017), Marrocos (El Khadir et al., 2017) e Colômbia (Melo-Narvaez et al., 2018), onde as cepas de $H$. pylori vacA do tipo il foram mais frequentes e associadas a uma maior prevalência em pacientes com desfechos clínicos graves.

Além das cepas de $H$. pylori que carregavam o genótipo vacA s1/m1 e s2/m2 serem i1 e i2 respectivamente, em um estudo realizado em Bangladesh, foi descoberto que o genótipo mais virulento i1/d1/c1 estava fortemente associado a escores histológicos graves do que em cepas com o genótipo i $2 / \mathrm{d} 2 / \mathrm{c} 2$. Os autores confirmaram nesse estudo que a região-m do vacA estava independentemente associada a um risco maior de atrofia no antro, mediante o ajuste para idade, sexo e status do cagA (Aftab et al., 2017).

Já em um estudo realizado na Polônia, foi observada a relação da expressão genética do vacA nos desfechos clínicos. $\mathrm{O}$ vacA apresentou atividade moderada em cepas de H. pylori isoladas de pacientes com gastrite não atroférica e maior atividade nos isolados com gastrite atrófica multifocal e câncer gástrico (Szkaradkiewicz et al., 2016).

Por outro lado, Akeel et al. (2019), mostraram que os alelos de vacA s1a/ m1, s1b/m1 e m1 eram os subtipos menos prevalentes e os alelos vacA s1a/ m1, s1a/m2 e s1b/m2 foram associados a úlcera gástrica e úlcera duodenal. Em concordância com este estudo, Pajavand et al. (2015), relataram que os subtipos predominantes de vacA em seu estudo eram: $\mathrm{s} 1 / \mathrm{m} 1$ e s $2 / \mathrm{m} 2$, com frequência de $39,5 \%$ e $50 \%$, respectivamente, enquanto o subtipo menos frequente era o s1/m1 com frequência de $7 \%$.

Já em um estudo realizado por Saeidi et al (2017), no Irã, eles encontraram que todas as cepas de $H$. pylori carregavam o gene vacA e a avaliação dos genótipos de vacA determinou o genótipo s1/m2 como o mais prevalente em pacientes com úlcera péptica.

\subsubsection{Associações entre cagA e vacA}

Diversas cepas de H. pylori apresentam combinações de alelos distintos e a coexistência de um ou mais desses genes resultam em um aumento na severidade das mudanças histopatológicas. A compreensão destas informações são importantes, pois elucidam a existência de linhagens mais virulentas de $H$. pylori, e que a combinação de cagA e vacA sejam uma das formas de avaliar o potencial patogênico da cepa de H.pylori (Leyya, Aleaga, González, Zamora \& González, 2016).

A combinação alélica mais frequente observada nos estudos foi cagA positivo e vacA s1/m1; este resultado apoia o consenso de que o genótipo vacA s1 está ligado ao status de positividade do cagA. Linhagens que apresentaram este genótipo foram consideradas mais virulentas e associadas a desfechos clínicos graves como: gastrite atrófica, úlceras pépticas, metaplasia e câncer gástrico (Molina-Castro, Garita-Cambronero, Malespin-Bendaña, Une \& Ramírez, 2019; Melo-Narvaez et al., 2018; Archampong et al., 2017; Idowu et al., 2019 Tserentogtokh et al., 2019; Pinto-Ribeiro et al., 2016). 
Em um estudo realizado no Brasil, foram observadas cepas virulentas com a combinação cagA positivo e vacA s1/m1. Este genótipo foi observado em cepas de H. pylori isoladas de pacientes com úlceras gástrica, úlcera duodenal e câncer gástrico, também foi observado que os pacientes infectados por estas cepas apresentaram alto grau de inflamação e atividade neutrofílica na mucosa gástrica, bem como um risco maior de desenvolvimento de metaplasia intestinal (Vinagre, Queiroz, Silva Júnior, Vinagre \& Martins, 2015).

Um resultado semelhante pode ser observado em um estudo em Okinawa, no Japão, onde as cepas de $H$. pylori que carregavam o genótipo vacA s1/i1/m1 e genes cagA do tipo leste asiático tinham uma forte associação com o câncer gástrico e úlceras gástricas (Inagaki et al 2017).

Sheikh et al. (2018), em um estudo realizado no Irã, observaram que cepas cagA positivas combinadas com vacA s1/m1 eram mais comuns em pacientes com câncer gástrico e úlcera péptica, enquanto, a combinação alélica com vacA s1/m2 foi mais predominante em pacientes com dispepsia não ulcerativa e gastroduodenite erosiva.

Um outro resultado pode ser observado no estudo de Pajavand et al. (2015) também realizado no Irã, onde a combinação cagA positivo e o genótipo vacA s1/m2 foi significativamente correlacionada com a úlcera péptica. Porém, o desenvolvimento de gastrite associado à infecção por $H$. pylori foi marcado por cepas que carregavam os genótipos cagA positivo e vacA s2m2, e esta foi a mais prevalente na população estudada.

Curiosamente neste mesmo estudo, das seis cepas consideradas cagA negativas, três cepas carregavam o genótipo vacA s1/m2 e foram associadas a úlcera péptica e as outras três cepas carregavam o genótipo s1/m1 foram relacionadas à gastrite (Pajavand et al., 2015).

Além destes genótipos existem cepas de $H$. pylori que apresentam o genótipo cagA negativo e vacA s2/i2/m2 que são classificadas como menos virulentas ou não virulentas, um achado que foi observado em um estudo realizado no Marrocos onde esta combinação foi a mais prevalente, o que justificou a baixa incidência de desfechos clínicos graves na população (El khadir et al., 2017).

\section{Conclusão}

Esta revisão sugere que os polimorfismos existentes nos fatores de virulência CagA e VacA, possuem uma forte associação com diversos desfechos clínicos gastroduodenais, e que a frequência/ gravidade da doença gastroduodenal associada ao $H$. pylori varia geograficamente. Porém, mesmo existindo uma grande quantidade de estudos clínicos/epidemiológicos com relação a esta temática, ainda é necessário termos cuidados ao tirar conclusões sobre as associações destes fatores de virulência e o desenvolvimento de patologias gastroduodenais. Pois, existem alguns resultados conflitantes e que a maioria dos estudos realizados avaliaram um pequeno número de pessoas infectadas e de cepas de $H$. pylori.

E por isso, a compreensão destes achados é de extrema importância, para identificação precoce de pacientes infectados por genótipos mais virulentos auxiliando no manejo do tratamento, pois a falta de tratamento ou falha na terapêutica, podem expor estes indivíduos ao desenvolvimento de desfechos clínicos gastroduodenais mais graves, como úlceras e câncer.

Com isso, a identificação dos diversos fatores de virulência, assim como o cagA e vacA, em pacientes infectados por H. pylori podem servir como marcadores moleculares epidemiológicos, que possam ser utilizados no desenvolvimento de futuras estratégias de prevenção e podem oferecer uma avaliação prognóstica individualizada, levando a um tratamento e gerenciamento mais personalizado dos pacientes que sofrem de infecções associadas por H. pylori. 


\section{Referências}

Aftab, H., Miftahussurur, M., Subsomwong, P., Ahmed, F., Khan, A. K. A., Matsumoto, T., Suzuki, R., \& Yamaoka, Y. (2017). Two populations of lessvirulent Helicobacter pylori genotypes in Bangladesh. Plos One, 12(8).

Akeel, M., Shehata, A., Elhafey, A., Elmakki, E., Aboshouk, T., Ageely, H., \& Mahfouz, M. (2019). Helicobacter pylori vacA, cagA and iceA genotypes in dyspeptic patients from southwestern region, Saudi Arabia: distribution and association with clinical outcomes and histopathological changes. BMC Gastroenterology, 19 (1), 16.

Ansari S, Yamaoka Y. (2019). Helicobacter pylori Virulence Factors Exploiting Gastric Colonização e sua Patogenicidade. Toxin as (Basileia).11(11):677.

Archampong, T. N., Asmah, R. H., Aidoo, E. K., Wiredu, E. K., Gyasi, R. K., Adjei, D. N., Beleza, S., Bayliss, C. D., \& Krogfelt, K. (2017). Helicobacter pylori cagA and vacA genes in dyspeptic Ghanaian patients. BMC Research Notes, 10(1), 231.

Backert, S., Blaser, M. J. (2016). The Role of CagA in the Gastric Biology of Helicobacter pylori. Cancer Res. 76(14), 4028-4031.

Bibi, F., Alvi, S. A., Sawan, S. A., Yasir, M., Sawan, A., Jiman-Fatani, A. A., \& Azhar, E. I. (2017). Detection and Genotyping of Helicobacter pylori among Gastric ulcer and Cancer Patients from Saudi Arabia. Pakistan journal of medical sciences, 33(2), 320-324.

Diaconu, S., Predescu, A., Moldoveanu, A., Pop, C. S., \& Fierbințeanu-Braticevici, C. (2017). Helicobacter pylori infection: old and new. J Med Life. $10(2): 112-117$

El Khadir, M., Alaoui Boukhris, S., Benajah, D. A., El Rhazi, K., Ibrahimi, S. A., El Abkari, M., Harmouch, T., Nejjari, C., Mahmoud, M., Benlemlih, M., \& Bennani, B. (2017). VacA and CagA Status as Biomarker of Two Opposite End Outcomes of Helicobacter pylori Infection (Gastric Cancer and Duodenal Ulcer) in a Moroccan Population. PloS one, 12(1).

Foegeding, N. J., Caston, R. R., McClain, M. S., Ohi, M. D., \& Cover, T. L. (2018). An Overview of Helicobacter pylori VacA Toxin Biology. Toxins. 8(6), 173-194.

Hatakeyama, M. (2017). Structure and function of Helicobacter pylori CagA, the first-identified bacterial protein involved in human cancer. Proc Jpn Acad Ser B Phys Biol Sci. 93(4):196-219.

Hooi, J. K. Y., et al (2017). Global Prevalence of Helicobacter pylori Infection: Systematic Review and Meta-Analysis. Gastroenterology. 153(2):420-429.

Idowu, A., Mzukwa, A., Harrison, U., Palamides, P., Haas, R., Mbao, M., Mamdoo, R., Bolon, J., Jolaiya, T., Smith, S., Ally, R., Clarke, A., \& Njom, H. (2019). Detection of Helicobacter pylori and its virulence genes (cagA, dupA, and vacA) among patients with gastroduodenal diseases in Chris Hani Baragwanath Academic Hospital, South Africa. BMC gastroenterology, 19(1), 73.

Imkamp, F., Lauener, F. N., Pohl, D., Lehours, P., Vale, F. F., Jehanne, Q., Zbinden, R., Keller, P. M., \& Wagner, K. (2019). Rapid Characterization of Virulence Determinants in Helicobacter pylori Isolated from Non-Atrophic Gastritis Patients by Next-Generation Sequencing. Journal of clinical medicine, 8(7), 1030 .

Inagaki, T., Nishiumi, S., Ito, Y., Yamakawa, A., Yamazaki, Y., Yoshida, M., \& Azuma, T. (2017). Associations Between CagA, VacA, and the Clinical Outcomes of Helicobacter Pylori Infections in Okinawa, Japan. The Kobe journal of medical sciences, 63(2), E58-E67.

Kao, C. Y., Sheu, B. S., \& Wu, J. J. (2016). Helicobacter pylori infection: An overview of bacterial virulence factors and pathogenesis. Biomed J. 39(1):14-23.

Leyva, L. M., Aleaga, Y. M., González, B. L. R., Zamora, O. R., \& González, S. R. M. (2016). Presencia del gen cagA y de la citotoxina vacA del Helicobacter pylori en pacientes dispépticos. Revista Cubana de Medicina Militar, 45(4), 1-9.

McClain, M. S., Beckett, A. C., \& Cover, T. L. (2017). Helicobacter pylori Vacuolating Toxin and Gastric Cancer. Toxins (Basel). 9(10), 316.

Melo-Narváez, M. C., Rojas-Rengifo, D. F., Jiménez-Soto, L. F., Delgado, M. d. P., Molano, B. M. d., Vera-Chamorro, J. F., \& Jaramillo, C. (2018). Genotyping of cagA and the intermediate region of vacA in strains of Helicobacter pylori isolated from Colombian adult patients and associations with gastric diseases. Rev Colomb Gastroenterol, 33(2), 103-110.

Mendes, K. D. S., Silveira, R. C. C. P., \& Galvão, C. M. (2008). Revisão integrativa: método de pesquisa para a incorporação de evidências na saúde e na enfermagem. Texto \& Contexto Enfermagem. 17(4): 758-764.

Molina-Castro, S., Garita-Cambronero, J., Malespín-Bendaña, W., Une, C., \& Ramírez, V. (2019). Virulence factor genotyping of Helicobacter pylori isolated from Costa Rican dyspeptic patients. Microbial pathogenesis, 128, 276-280.

Pajavand, H., Alvandi, A., Mohajeri, P., Bakhtyari, S., Bashiri, H., Kalali, B., Gerhard, M., Najafi, F., \& Abiri, R. (2015). High Frequency of vacA s1m2 Genotypes Among Helicobacter pylori Isolates From Patients With Gastroduodenal Disorders in Kermanshah, Iran. Jundishapur journal of microbiology, 8(11), e25425.

Paredes-Osses, E., Sáez, K., Sanhueza, E., Hebel, S., González, C., Briceño, C., \& García Cancino, A. (2017). Association between cagA, vacAi, and dupA genes of Helicobacter pylori and gastroduodenal pathologies in Chilean patients. Folia microbiologica, 62(5), 437-444.

Pinto-Ribeiro, I., Ferreira, R. M., Batalha, S., Hlaing, T., Wong, S. I., Carneiro, F., \& Figueiredo, C. (2016). Helicobacter pylori vacA Genotypes in Chronic Gastritis and Gastric Carcinoma Patients from Macau, China. Toxins, 8(5), 142.

Saeidi, Y., Pournajaf, A., Gholami, M., Hasannejad-Bibalan, M., Yaghoubi, S., Khodabandeh, M., Emadi, B., Ferdosi-Shahandashti, E., \& Rajabnia, R. (2017). Determination of Helicobacter pylori virulence-associated genes in duodenal ulcer and gastric biopsies. Medical journal of the Islamic Republic of Iran, 31, 95. 
Research, Society and Development, v. 10, n. 4, e28610412938, 2021

(CC BY 4.0) | ISSN 2525-3409 | DOI: http://dx.doi.org/10.33448/rsd-v10i4.12938

Sheikh, A. F., Yadyad, M. J., Goodarzi, H., Hashemi, S. J., Aslani, S., Assarzadegan, M. A., \& Ranjbar, R. (2018). CagA and vacA allelic combination of Helicobacter pylori in gastroduodenal disorders. Microbial pathogenesis, 122, 144-150.

Souza, M. T., Silva, M. D.,\& Carvalho, R. (2010). Revisão Integrativa: o que é e como fazer. Einstein, 8(1), $102-106$.

Sterbenc, A., Jarc, E., Poljak, M., \& Homan, M. (2019). Helicobacter pylori virulence genes. World Journal of Gastroenterology. 25(33), 4870-4884.

Szkaradkiewicz, A., Karpiński, T. M., Linke, K., Majewski, P., Rożkiewicz, D., \& Goślińska-Kuźniarek, O. (2016). Expression of cagA, virB/D Complex and/or vacA Genes in Helicobacter pylori Strains Originating from Patients with Gastric Diseases. PloS one, 11(2), e0148936.

Thi Huyen Trang, T., Thanh Binh, T., \& Yamaoka, Y. (2016). Relationship between vacA Types and Development of Gastroduodenal Diseases. Toxins. 8(6), 182-192.

Tserentogtokh, T., Gantuya, B., Subsomwong, P., Oyuntsetseg, K., Bolor, D., Erdene-Ochir, Y., Azzaya, D., Davaadorj, D., Uchida, T., Matsuhisa, T., \& Yamaoka, Y. (2019). Western-Type Helicobacter pylori CagA are the Most Frequent Type in Mongolian Patients. Cancers, 11(5), 725.

Vinagre, I. D., Queiroz, A. L., Silva Júnior, M. R., Vinagre, R. M., \& Martins, L. C. (2015). Helicobacter pylori infection in patients with different gastrointestinal diseases from northern brazil. Arquivos de gastroenterologia, 52(4), 266-271.

Whitmire, J. M., \& Merrell, D. S. (2019). Helicobacter pylori Genetic Polymorphisms in Gastric Disease Development. Adv Exp Med Biol. 